\title{
A Review \\ on Artificial Intelligence in Special Education
}

\author{
Athanasios S. Drigas and Rodi-Eleni Ioannidou \\ NCSR DEMOKRITOS, \\ Institute of Informatics and Telecommunications \\ Net Media Lab, \\ Ag. Paraskevi, 15310, Athens, Greece \\ drait.demokritos.gr, \\ elena.ioan@hotmail.com
}

\begin{abstract}
Innovative educational technologies have started to open new ways of interacting with students with special educational needs (SEN). Amongst the most effective approaches during the last decade (2001-2010) are those based on Artificial Intelligence (A.I.) techniques. The effective application of A.I. methods is seen as a means of improving the quality of life of SEN learners. Hence, a need for introducing A.I. techniques arises in order to develop both diagnosis and intervention processes. This paper presents a brief overview of the most representative studies of the past ten years, used for the above purposes.
\end{abstract}

Keywords: Artificial Intelligence, special educational needs, learning difficulties, diagnosis, intervention.

\section{Introduction}

The constant development in technology has dramatically transformed the world during the past decade and devolved computing power in every aspect of our daily life. One of the most important aspirations of computing science was the quest to understand human intelligence in all forms. This quest for the last fifty years led a large number of researchers to develop the field of Artificial Intelligence (A.I.). A.I. is usually defined as the study and design of intelligence agents which are able to perceive their environment and take actions that increase their possibilities of success [1].

One of the research communities of Artificial Intelligence field deals with the intersection of A.I. and education. The vast majority of scientists and researchers seem to support the idea that A.I. tools can successfully contribute to the educational process [2]. Recently there has been an increasing emphasis on educating all children and ensuring the learning needs of all young people and adults. This fact has reinforced the use of technology as a mean of overcoming barriers to learning. The benefits of A.I. in education have been lauded for many years. Artificial Intelligence methods have long been used to the field of special education as well. The first 
research tasks mainly applied 'Expert Systems' of which the only aim was to model the behavior of a human expert to achieve an intellectual task [3].

The term 'special educational needs' refers to a wide range of difficulties which cause problems in learning. In addition, it has much to do with the cultural and historical development of research in different countries. Since a term such as 'special educational needs' is very general, for the needs of this paper we decided to use the definition of 'learning difficulties' which is closer to the one of 'learning disabilities', as it is defined in IDEA. The 'Individuals with Disabilities Education Act' (IDEA) defines learning disability as a 'disorder in one or more of the basic psychological processes involved in understanding or in using spoken or written language, which may manifest itself in an imperfect ability to listen, think, speak, read, write, spell or to do mathematical calculations' [3].

Link-up between Artificial Intelligence educational approaches and students with different abilities and needs has opened new eras and trends which require use of specific tools and new methods in order to improve children's life in both school and home settings. During the last decade there have been an important number of studies which address how A.I. computing tools can be used in practice in order to improve the quality of education of students with learning difficulties [4]. In this article we will focus on the most representative Artificial Intelligence techniques of the last decade which have been used for the diagnosis and intervention processes of learners with special educational needs. These assistive tools can be used from school staff, special educators and parents as well. In order for these studies to be classified, this report consists of two main categories. In the first one we introduce applications regarding the identification of learning difficulties and the second section includes intervention based tools.

\section{Diagnosis}

Considering the implicit characteristics of learning difficulties, the diagnosis with A.I. methods of learners with learning difficulties has long been an issue with great debate around it [5]. The variety of symptoms and nature of the special educational needs, the co-occurrence with other disorders, the differences between boys and girls are some of the several issues that arise during the assessment procedure.

Georgopoulos et al., 2003 presented a fuzzy cognitive map approach for differential diagnosis of specific language impairment (SLI). Fuzzy cognitive maps are a soft computing methodology that uses a symbolic representation for the description and modeling of complex systems. The aim of this tool is to provide the specialists with a differential diagnosis of SLI from dyslexia and autism, since in many cases SLI is difficult to be discerned due to its similar symptoms to other disorders. The system has been tested on four clinical cases with promising results [6].

In 2004 Rebolledo-Mendez and Freitas presented the NeuroSky MindSet (MS) which is able to detect attention levels in an assessment exercise by combining performance data with user-generated data, taken from interaction. NeuroSky consists of a headset with three electrodes, which are put beneath the ears and on the forehead. The electrical signals read at the above locations are used as inputs by NeuroSky's 
algorithms to assess the attention levels. An A.I. driven avatar was also designed to pose questions and have limited conversation with the users. It is a low-cost, nonclinical and easy to use tool designed for leisure. This model was tested on first-year undergraduate students in the following years and the results indicated that there is a positive relation between measured and self-reported levels of attention [7] , [8].

Arthi and Tamilarasi (2008) introduced a model which helps in the diagnosis of autism in children by applying Artificial Neural Networks (ANN) technique. The model converts the original autistic data into suitable fuzzy membership values and these are given as input to the neural network architecture. Moreover, a pseudo algorithm is created for applying back propagation algorithm in predicting the autistic disorder. This approach is proposed to support apart from medical practitioners, psychologists and special educators. In future the autistic disorder could be predicted using k-nearest neighbor algorithm for a comparative research [9].

Hernández et al., (2009) introduced SEDA ('Sistema Experto de Dificultades para el Aprendizaje' or 'Expert System for Learning Difficulties' in English) a diagnostic tool for Learning Difficulties in children's basic education. It is developed using the Expert Systems design methodologies which include a knowledge base consisting of a series of strategies for Psycopedagogy evaluation; trying to identify the relationships between input variables (e.g. age, sex, educational level) and the output systems (e.g. psychomotor aspect, intellectual aspect). All of the above provides the expert system's users the possibility of acknowledge the psychological profile of the pupil. $80 \%$ of the evaluators rated the system as Efficient using an estimation scale of: Poor, Moderately Efficient and Efficient [10], [11].

In the same year Jain et al., (2009) proposed a model called Perceptron based Learning Disability Detector (PLEDDOR). It is an artificial neural network model for identifying difficulties in reading (dyslexia), in writing (dysgraphia) and in mathematics (dyscalculia) using curriculum based test conducted by special educators. This computational diagnostic tool consists of a single input layer with eleven units that correspond to different sections of a conventional test and one output unit. The system was tested on 240 children collected from schools and hospitals in India and was evaluated as simple and easy to replicate in huge volumes, but provides comparable results based on accepted detection measures [12].

Kohli et al., (2010) introduced a systematic approach for identification of dyslexia at an early stage by using artificial neural networks (ANN). This approach is amongst the first attempts which have been made for addressing the dyslexia identification problems with the use of ANN. Moreover, it can be distinguished from other platforms of its kind because it is based on test data, covering the evaluation results of potential dyslexic pupils, between the years 2003 - 2007. These test data consist the input data of the system while the output results classify the students in two categories (dyslexic and non-dyslexic). An error back-propagation algorithm is responsible for mapping college performance to the underlying characteristics. The initial results obtained using test data were fairly accurate and suggest the application of this platform to real data as well [13].

Anuradha et al., (2010) developed a platform for a more accurate and less time consuming diagnosis of Attention Deficit Hyperactivity Disorder (ADHD). They used one well-known Artificial Intelligence technique, the SVM algorithm. According to the authors, this is the first attempt at identifying ADHD using SVM algorithm. 
Support vector machines (SVMs) are a set supervised learning techniques suitable for classification and regression. A data-set which was verified by a doctor including the results of a questionnaire used by the doctors to diagnose the disorder was given to the SVM module. After that the data-set was introduced and afterwards returned to the SVM module, which finally provides us with the diagnosis. The most important advantage of applying the SVM algorithm is that it can control the complexity of the diagnostic process. This method was tested on children between the ages six to eleven years old and the results indicated a percentage of $88,674 \%$ success in diagnosing [14].

\section{Intervention}

A reliable and valid diagnosis is the first step in order to help a child overcome his or her difficulties. The second step is the intervention process. An important number of studies are currently addressing the use of Artificial Intelligence systems in the education of students with learning difficulties.

Melis et al., (2001) introduced ActiveMath, a web-based intelligent tutoring system for mathematics. ActiveMath is an Intelligence Tutoring System (ITS), which allows the students to learn in their own environment whenever it is convenient for them. It uses a number of Artificial Intelligence techniques to realize adaptive course generation, student modeling, feedback, interactive exercises and a knowledge representation which is appropriate for the semantic Web. In ActiveMath the user starts his/her own student model by self-assessment of his/her mastering level of concepts and later chooses learning goals and scenario, for instance, the preparation for an exam. The capabilities of the student are adapted in course generation and in the suggestion mechanism as well. Moreover, a "poor man's eye-tracker" is designed which is able to trace child's attention and reading time in detail. This application has reported many positive outcomes in the following years by a large number of studies, all of them supporting the effect of this ITS during the learning process [15], [16], [17].

Schipor et al., (2003) attempted to create a Computer Based Speech Therapy (CBST) system using a fuzzy expert system for helping learners with speech disorders. They designed an improved CBST system, called LOGOMON (Logopedics Monitor) and developed its classical architecture with a fuzzy expert system. The aim of this approach is to suggest optimal therapeutic actions for every pupil based on the information selected. The evaluation of this system indicates that by using an expert system, the learner is provided with more therapy time, predictability and the explanation of results [18].

In 2007 Riedl et al, designed a platform which can aid adolescents with High Functioning Autistic Spectrum Disorders (HFASD) rehearse and learn social skills with reduced help from parents, teachers, and therapists.. A social scenario game is presented - for example going to a movie theater- which challenges learners with HFASD to role-play and complete tasks involving social situations. Artificial Intelligence is used to assist the above groups with the authoring of tailored social scenarios. An A.I. system automatically examines the causal form of the narrative plan, searching for points at which a student's actions can undo causal relationships. 
The alternative narrative scenario is a branch developed for handling the contingency of the learner's action. This Artificial Intelligence tool embed in this particular platform decreases the manual authoring burden where application of intervention strategies can be handled by specialists. This social scenario intervention approach is complete and currently undergoing evaluation with promising results [19].

In 2008 Drigas et al., presented 'Dedalos' project which deals with the teaching of the English language as a second language to hearing impaired people, whose mother language is the Greek sign language. In an educational e-content adapted to the needs of every user, the whole procedure consists of audits and evaluation of the linguistic abilities of the e-learners. The system uses an intelligence taxonomy system which is developed for the evaluation of the pupil and the setting of pedagogic material. The approach promotes a complete support system for the education of hearing impaired Greek students while at the same time opens the way for their inclusion [20].

Gonzalez et al, (2010) designed an automatic platform for the detection and analysis of errors in mathematical problems to support the personalized feedback of pupils. This method is referred to all students and particularly to students with special educational needs such as those with Down syndrome, who exhibit difficulties in the arithmetic operations of addition and subtraction. An error detection algorithm was developed which is able to analyze the data gathered as a result of the interaction between the students and the platform, while afterwards the output of the error is available to the teachers about the specific difficulties and to allow them to personalize the instruction. Moreover, they designed a model which returns the set of errors made by the pupils in the corrected exercises so as the students can learn from their own mistakes. The system was tested on a group of students with Down syndrome and the results confirm that the module exhibits the proper behavior [21].

In the same year Baschera and Gross introduced an adaptive spelling training system which can be used from all students who exhibit spelling difficulties. This platform is based on an inference algorithm designed to manage unclassified input with multiple errors defined by independent mal-rules. The inference algorithm based on a Poisson regression with a linear link function, estimates the pupil's difficulties with each individual mal-rule, based on the observed error behavior. This knowledge representation was implemented in a student model for spelling training such as optimized word selection and lessons for individual mal-rules to pupil adjusted repetition of erroneously spelled words. This system was tested on a two large-scale user studies and showed an important increase in the learner's performance, induced by the student adapted training actions [22].

\section{Conclusions}

Artificial Intelligence techniques have successfully been applied to solve problems in the field of special education. There is a general consensus amongst scientists that Artificial Intelligence methods are able to integrate the freedom of action of the user and lead him or her toward personnel learning goals. This study has reported some of the most representative articles over the last decade which introduced diagnostic and intervention approaches based on Artificial Intelligence techniques. These educational tools are specifically oriented to assisting students with learning difficulties as well as 
teachers, parents, special educators and therapists. The identification of students with learning difficulties using A.I. techniques can provide us a valid and accurate diagnosis which later can help us choose the most appropriate intervention method. However there are still several issues which demand further research such as the lack of the offered A.I. intervention tools and the lack of nationally regulated standards regarding the A.I. diagnostic methods. Moreover, the future studies should include a larger sample of pupils and also groups of pupils with other learning difficulties. Research studies regarding the use of Artificial Intelligence technology are very promising and can ensure a better quality of the life of special educational needs students and all the people around them.

\section{References}

1. Russell, S.J., Norvig, P.: Artificial Intelligence: A Modern Approach, 2nd edn., New Jersey (2003)

2. Lanzilotti, R., Roselli, T.: An Experimental Evaluation of Logiocando, an Intelligent Tutoring Hypermedia System. International Journal of Artificial Intelligence in Education 17, 41-56 (2007)

3. Wu, T.K., Meng, Y.R., Huang, S.C.: Application of Artificial Neural Network to the Identification of Students with Learning Disabilities. In: International Conference on Artificial Intelligence, pp. 162-168 (2006)

4. Public Law, Individuals with Disabilities Education Act (IDEA), USA, pp. 101-476 (1990)

5. Nanni, L., Lumini, A.: Ensemble generation and feature selection for the identification of students with learning disabilities. Expert Systems with Applications 36, 3896-3900 (2008)

6. Georgopoulos, V.C., Malandraki, G.A., Stylios, C.D.: A fuzzy cognitive map approach to differential diagnosis of specific language impairment. Artificial Intelligence in Medicine 29, 261-278 (2003)

7. Rebolledo-Mendez, G., De Freitas, S.: Attention modeling using inputs from a Brain Computer Interface and user-generated data in Second Life. In: The Tenth International Conference on Multimodal Interfaces (ICMI 2008), Crete, Greece (2008)

8. Rebolledo-Mendez, G., Dunwell, I., Martínez-Mirón, E.A., Vargas-Cerdán, M.D., de Freitas, S., Liarokapis, F., García-Gaona, A.R.: Assessing NeuroSky's Usability to Detect Attention Levels in an Assessment Exercise. In: Jacko, J.A. (ed.) HCI International 2009, Part I. LNCS, vol. 5610, pp. 149-158. Springer, Heidelberg (2009)

9. Arthi, K., Tamilarasi, A.: Prediction of autistic disorder using neuro fuzzy system by applying ANN technique. International Journal of Developmental Neuroscience 26, 699704 (2008)

10. Hernadez, J., Mousalli, G., Rivas, F.: Expert System for the Diagnosis of Learning Difficulties in Children's Basic Education. In: Proceedings of the 8th WSEAS International Conference on Applied Computer and Applied Computational Science, Italy (2008)

11. Hernadez, J., Mousalli, G., Rivas, F.: Learning Difficulties Diagnosis for Children's Basic Education using Expert Systems. WSEAS Transactions on Information Science and Applications 7(6) (2009) 
12. Jain, K., Manghirmalani, P., Dongardive, J., Abraham, S.: Computational Diagnosis of Learning Disability. International Journal of Recent Trends in Engineering 2(3) (2009)

13. Kohli, M., Prasad, T.V.: Identifying Dyslexic Students by Using Artificial Neural Networks. In: Proceedings of the World Congress on Engineering, London, U.K, vol. 1 (2010)

14. Anuradha, J., Tisha, Ramachandran, V., Arulalan, K.V., Tripathy, B.K.: Diagnosis of ADHD using SVM algorithm. In: Proceedings of the Third Annual ACM Bangalore Conference (2010)

15. Melis, E., Andres, E., Budenbender, J., Frischauf, A., Goguadze, G., Libbrecht, P., Pollet, M., Ullrich, C.: ACTIVEMATH, A Generic and Adaptive Web-Based Learning Environment. International Journal of Artificial Intelligence 24(4), 1-25 (2001)

16. Libbrecht, P., Melis, E.: Methods to Access and Retrieve Mathematical Content in ActiveMath. In: Iglesias, A., Takayama, N. (eds.) ICMS 2006. LNCS, vol. 4151, pp. 331-342. Springer, Heidelberg (2006)

17. Melis, E., Siekmann, J.: ACtiveMath: An Intelligent Tutoring System for Mathematics. In: Rutkowski, L., Siekmann, J.H., Tadeusiewicz, R., Zadeh, L.A. (eds.) ICAISC 2004. LNCS (LNAI), vol. 3070, pp. 91-101. Springer, Heidelberg (2004)

18. Schipor, O.A., Pentiuc, S.G., Schipor, M.D.: Improving computer based speech therapy using a fuzzy expert system. Computing and Informatics 22, 1001-1016 (2003)

19. Riedl, M., Arriaga, R., Boujarwah, F., Hong, H., Isbell, J., Heflin, L.J.: Graphical Social Scenarios: Toward Intervention and Authoring for Adolescents with High Functioning Autism. In: Virtual Healthcare Interaction, Papers from the AAAI Fall Symposium (2007)

20. Drigas, A.S., Kouremenos, D., Vrettaros, J.: Teaching of English to Hearing Impaired Individuals Whose Mother Language Is the Sign Language. In: Lytras, M.D., Damiani, E., Tennyson, R.D. (eds.) WSKS 2008. LNCS (LNAI), vol. 5288, pp. 263-270. Springer, Heidelberg (2008)

21. Gonzalez, C.S., Guerra, D., Sanabria, H., Moreno, L., Noda, M.A., Bruno, A.: Automatic system for the detection and analysis of errors to support the personalized feedback. Expert Systems with Applications 37, 140-148 (2010)

22. Baschera, G.M., Gross, M.: Poisson-Based Inference for Perturbation Models in Adaptive Spelling Training. International Journal of Artificial Intelligence in Education 20, 1-31 (2010) 